\title{
Die Tuberkulose, vom Altertum in die Neuzeit
}

Einige Infektionskrankheiten haben sich unwiderruflich in das kollektive Bewusstsein der Menschen eingebrannt. Neben den Pocken und der Pest gehört zweifelsohne auch die Tuberkulose dazu. Auch wenn heute nur die wenigsten Menschen in den Industrienationen in Kontakt mit Tuberkuloseerkrankten kommen, hat die Krankheit kaum etwas von ihrem Schrecken verloren. Die Tuberkulose ist ein treuer Begleiter in der Menschheitsgeschichte. Sie war bereits in vorchristlicher Zeit präsent und ist immer noch verantwortlich für jährlich 1,7 Millionen Tote [1].

Roger M. Wanner, Burkhard Springer

Es bestehen keine Interessenverbindungen.

Korrespondenzen:

Dr. med. Roger M. Wanner Institut für medizinische Mikrobiologie

Gloriastrasse 30/32 CH-8006 Zürich

Tel. 0446342693

Fax 0446344906

rwanner@immv.uzh.ch

Dr. med Burkhard Springer Nationales Zentrum für Mykobakterien Universität Zürich Gloriastrasse 30/32 CH-8006 Zürich
Every man at the end has a little tuberculosis. Sir William Osler

\section{Das Krankheitsbild: von der Phtisis zur Tuberkulose}

Die Herkunft von Mycobacterium tuberculosis, dem Verursacher der Tuberkulose, wurde in letzter Zeit intensiv beforscht [2]. Es wird angenommen, dass sich einige Vertreter des Genus Mycobacterium ursprünglich im Erdboden, andere im Tierreich entwickelt hatten. Die Domestizierung von Rindern, die vor ungefähr 10000 bis 20000 Jahren stattfand [3], könnte die Übertragung des virulenten Bakteriums auf den Menschen ermöglicht haben.

Die Tatsache, dass Knochen Tausende von Jahren konserviert werden, ermöglichte den Nachweis von genetischem Material von Tuberkulosebakterien in Skeletten aus archäologischen Ausgrabungen [4] und in ägyptischen Mumien [5]. Trotzdem müssen diese Untersuchungen kritisch betrachtet werden, da immer die Gefahr von DNA-Kontaminationen aus dem 19. und 20 Jahrhundert besteht [6].

Wegen der Vielfalt an Symptomen und pathologischen Befunden dauerte es sehr lange, bis die Tuberkulose als eigenständige Krankheit identifiziert wurde. Hippokrates (etwa 460-370 v. Chr.) beschrieb Patienten mit Phtisis, die mit Brustschmerz und Husten, manchmal sogar mit blutigem Sputum vor sich hinsiechten [7]. Er zählte die Phtisis zu den häufigsten Krankheiten jener Zeit. Obwohl man damals glaubte, die Krankheit sei vererbt, warnte er seine Kollegen vor dem Besuch von Patienten, die in einem fortgeschrittenen Stadium der Schwindsucht litten. Galen von Pergamon (um 130-200) definierte die Phtisis als
Ulzeration der Lunge. 1500 Jahre später beschrieb der englische Arzt Richard Morton (1637-1698) zum ersten Mal weissliche Tuberkel in Autopsien von allen an Phtisis verstorbenen Patienten. In seiner Publikation «Phtisiologia, seu exercitationes de phtisi» [8] legte er dar, dass diese Tuberkel eine notwendige Bedingung für die Lungenerkrankung waren, die man später Tuberkulose nennen würde. René Théodophile Hyacinthe Laënnec (1781-1826) erkannte, dass die verschiedenen Veränderungen in der Lunge von PhtisisErkrankten unterschiedliche Stadien des gleichen pathologischen Prozesses waren und damit die Abgrenzung von einer Pneumonie erlaubten. 1839 gab der deutsche Arzt Johann Lukas Schönlein (1793-1864), der auch einige Jahre in Zürich lehrte, der Lungenkrankheit ihren Namen, den er von den typischen Läsionen in Autopsien, den Tuberkeln, ableitete [9].

\section{Der Erreger: vom Bacillus zu Mycobacterium tuberculosis}

Noch im 1881 publizierten Lehrbuch «The Principles and Practice in Medicine» von Flint und Welch stand, dass allgemein angenommen würde, dass die Tuberkulose nicht übertragbar sei. Ein Jahr später gelang Robert Koch (1843-1910) der Nachweis, dass die Tuberkulose eine bakterielle Erkrankung ist. Der Artikel von Koch «Die Aetiologie der Tuberculose» [10] lieferte neben dem Beweis für die Existenz des Tuberkelbazillus auch eine genaue Beschreibung der Diagnostik: die Färbemethode, die Herstellung von Reinkulturen und die Durchführung von Tierversuchen. Im gleichen Artikel stellte er die immer noch gültigen Koch'schen Postulate auf: «Um zu beweisen, dass die Tuberculose eine durch die Einwanderung der Bacillen veranlasste und in erster Linie 
durch das Wachsthum und die Vermehrung derselben bedingte parasitische Krankheit sei, mussten die Bacillen vom Körper isolirt, in Reinculturen so lange fortgezüchtet werden, bis sie von jedem etwa noch anhängenden, dem thierischen Organismus entstammenden Krankheitsprodukt befreit sind, und schliesslich durch Uebertragung der isolirten Bacillen auf Thiere dasselbe Krankheitsbild der Tuberculose erzeugt werden, welches erfahrungsgemäss durch Impfung mit natürlich entstandenen Tuberkelstoffen erhalten wird.» (dic). 1905 erhielt Koch für seine Untersuchungen und die Entdeckung des Tuberkuloseerregers den Nobelpreis.

\section{Die Therapie: von immunen Orten und Kollapstherapien}

Die Behandlungsansätze der Schwindsucht waren sehr vielfältig. Hermann Brehmer (18261889) litt im jugendlichen Alter unter Tuberkulose. Ein Aufenthalt im Himalaya befreite ihn von seinen Leiden. Nach dem Medizinstudium und seiner Dissertation «Die Gesetze und die Heilbarkeit der chronischen Tuberkulose der Lunge» eröffnete er an einem «immunen Ort», dem heute polnischen Göbersdorf, das erste Sanatorium für Tuberkuloseerkrankte. Er definierte unter einem «immunen Ort» eine Region, in der keine Schwindsüchtigen waren. Die ersten Sanatorien der Schweiz wurden 1895 in Heiligenschwendi bei Thun und 1886 in Davos eröffnet. Bereits zwanzig Jahre später zählte man in Davos zwölf kantonale und ausländische Sanatorien [11]. Die Heilstätten hatten zweierlei Funktion: Einerseits sollten sie die Bevölkerung vor Ansteckung schützen, andererseits ermöglichten sie den Patienten Bettruhe, frische Luft, viel Licht und gutes Essen.

Die Beweise für den Nutzen der «Dauer-Luftund Ruhe-Kur» - so die vollständige Bezeichnung der Freiluftliegekur - lieferten Edward Livingston Trudeau (1848-1915) und Niels Ryberg Finsen (1860-1904). Der amerikanische Arzt Trudeau zeigte, dass die Genesung von mit Tuberkeln infizierten Kaninchen besser auf einer grünen Insel ablief, als wenn man sie in ein dunkles und dreckiges Erdloch steckte, wo sie nur mit alten Kartoffeln gefüttert wurden. Er beschrieb, dass «the flesh [der auf der Insel ausgesetzten Tiere] was so firm and red as to be in striking contrast to the blanched and flabby muscles of the other rabbits» [12]. Der Däne Finsen erzielte mit Lichtstrahlen Behandlungserfolge gegen die Hauttuberkulose [13]. Für diese Entdeckungen erhielt er 1903 den Nobelpreis. Leider fehlen aus jener Zeit Studien, die die Letalität von Sanatoriumspatienten mit unbehandelten Tuberkulosepatienten verglichen.
Ende des 19. Jahrhunderts stellte der italienische Chirurge Carlo Forlanini (1847-1918) die sogenannte Kollapstherapie vor: «Wenn ein Pneumothorax den Verlauf der Phtisis in der Lunge stoppt, wieso sollten wir dann nicht bei Schwindsüchtigen durch die Thoraxwand einen künstlichen Pneumothorax herstellen?» [14]. Die Grundidee war, dass ein künstlicher Pneumothorax die Heilung im immobilisierten und damit stressfreien Gewebe fördert. Die fehlende Ventilation der betroffenen Lungenareale führte dazu, dass zwar im Sputum der Patienten keine Tuberkulosebakterien mehr nachgewiesen werden konnten, der Verlauf der Tuberkulose aber kaum beeinflusst wurde [15].

\section{Die Prophylaxe: \\ Fortschritt und Rückschlag}

1884 präsentierte Louis Pasteur (1822-1895) das Prinzip der aktiven Impfung gegen virulente Krankheiten. Er stellte aus lebenden Mikroben einen Impfstoff gegen die Tollwut her, den er 1885 an einem jungen Mann erfolgreich testete. 1906 gelang den beiden Mikrobiologen Albert Calmette (1863-1933) und Jean-Marie Camille Guérin (1872-1961) der Durchbruch zur Tuberkuloseprophylaxe. Sie nutzten Pasteurs Prinzip, um aus Mycobacterium bovis einen Impfstamm zu entwickeln: Nach 13 Jahren und 230 Passagen in Ochsengalle hatte $M$. bovis seine tödliche Wirkung in Meerschweinchen, Kaninchen, Kühen, Pferden und Schimpansen verloren [16]. Das so abgeschwächte Bakterium rief einen gewissen Schutz vor einer Infektion mit M. tuberculosis hervor. Einen grossen Rückschlag erlebte die BCGImpfung durch Impfschäden in Deutschland, die als «Lübecker Impfunglück» in die Geschichte eingegangen sind. 1930 wollten Ernst Altstaedt (1885-1953) und Georg Deycke (1865-1938) die in Frankreich breit verwendete Impfung in Deutschland einführen. Von 256 geimpften Neugeborenen (ungefähr $84 \%$ aller Geburten in Lübeck) starben 77 Impflinge und weitere 131 Kinder erkrankten an Tuberkulose. Die Ursache war eine Kontamination der BCG-Kulturen mit pathogenen Tuberkulosebakterien wegen fehlender räumlicher Trennung des Impflabors zum Diagnostiklabor. Die beiden Verantwortlichen wurden später wegen fahrlässiger Tötung und fahrlässiger Körperverletzung für schuldig befunden [17]. Daraufhin wurde in vielen europäischen Ländern, so auch in der Schweiz, die BCG-Impfung gestoppt und erst nach dem 2. Weltkrieg wieder eingeführt. Seither wurde die BCG-Impfung mehreren Milliarden Menschen verabreicht und gilt als der weltweit am häufigsten verwendete Impfstoff. Allerdings konnte der Impfschutz 
nicht generell nachgewiesen werden. Schliesslich war es ein von der WHO gross angelegter Feldversuch in Indien, bei dem 360000 Personen eingeschlossen wurden, der Klarheit schuf. Die gesamte Bevölkerung wurde mit BCG geimpft und 7,5 Jahre beobachtet mit dem ernüchternden Resultat: «The results of the trial show that BCG did not give any protection during the first $7 \frac{1}{2}$ years after vaccination.» [18]

\section{Antibiotika: Erfolg und Ernüchterung}

Der wahre Durchbruch in der Tuberkulosetherapie gelang 1944 Selman Abraham Waksman (1888-1973) mit der Entdeckung des Streptomycins. Streptomycin zeigte antibiotische Aktivität gegen 22 verschiedene Bakterien, darunter auch Mycobacterium tuberculosis [19]. Für diese Entdeckung erhielt Waksman 1952 den Nobelpreis. Bereits 15 Monate später und noch während Experimente an Meerschweinchen durchgeführt wurden, begann Karl Pfütze an der amerikanischen Mayo Clinic mit der Behandlung von Menschen: Während zweier Jahre erhielt eine 21-jährige Tuberkulosepatientin Streptomycin. Sie überlebte und galt als geheilt, war zehn Jahre später gesund [20, 21]. Auch in den Schweizer Sanatorien wurde das neue Heilmittel eingesetzt: 1948 dokumentierte Ernesto-Rubino Mordasini einen erfolgreich behandelten Fall mit Röntgenbildern [22]. Der Siegeszug des neuen Antibiotikums zeigte sich am Interesse der pharmakologischen Industrie: Von einem Laborkuriosum wuchs die industrielle Streptomycinproduktion allein in den USA auf 25 Tonnen pro Monat an. Obwohl Streptomycin sehr wirksam war, war es weit entfernt vom perfekten Medikament: Es hatte viele Nebenwirkungen, und M. tuberculosis konnte ziemlich rasch resistent werden. 1951 entdeckten drei Pharmafirmen (Bayer Chemical, Squibb und Hoffmann-La Roche) gleichzeitig Isoniazid. Diese Substanz war sehr wirksam, gut verträglich und billig. Bereits ein Jahr später wurde Pyrazinamid entdeckt. Seither wurden nur noch wenige neue Antituberkulostatika entdeckt: 1961 Ethambutol und 1966 Rifampicin. Diese vier Antibiotika sind immer noch die First-Line-Medikamente in der Behandlung der Tuberkulose [23].

Dank der antibiotischen Therapie konnte die Tuberkulose zwar nicht vollständig erradiziert, doch zumindest kontrolliert werden, bis 1985 die HIV-Epidemie zu einer starken Zunahme an Tuberkulosefällen führte.

Obwohl das Verständnis der Ätiologie und der Pathogenese seit der Entdeckung des Erregers vor 125 Jahren stark zugenommen hat, bleibt die
Tuberkulose eine weltweite Pandemie: Ein Drittel der Weltbevölkerung ist mit M. tuberculosis infiziert. Beinahe neun Millionen Neuinfektionen wurden 2004 geschätzt. 59\% der Tuberkulosefälle betreffen Patienten in unterentwickelten Ländern, in denen der Zugang zu Medikamenten und einer adäquaten Therapie schlecht ist und sich HIV und M. tuberculosis gegenseitig begünstigen. Die Tuberkulose kann geheilt werden, trotzdem sterben täglich 5000 Menschen an ihr [1].

In der Schweiz ist die Inzidenz der Tuberkulose seit Jahrzehnten rückläufig, mit einer aktuellen Inzidenz von 8 Fällen pro 100000 Einwohner (www.who.int/GlobalAtlas/dataQuery/default. asp) liegt die Schweiz im guten Mittelfeld der westlichen Industriestaaten. Der Ausländeranteil betrug dabei im Jahr 2004 64\% [24]. Aus dieser Zahl wird deutlich, dass sich die Schweiz nicht von der globalen Entwicklung abkoppeln kann. Das Auftreten von MDR- (multidrug resistant; definitionsgemäss resistent gegen Isoniazid und Rifampicin) und kürzlich beschriebenen XDRM. tuberculosis-Isolaten (extensively drug resistant; MDR plus Resistenz gegen Gyrasehemmer und eines der folgenden Antibiotika: Capreomycin, Kanamycin oder Amikacin) stellt eine neue Herausforderung auch für Industrienationen dar [25]. Um nicht in das Zeitalter der Sanatorien und chirurgischen Interventionen zurückzufallen, sind dringend neue Therapieoptionen und die Entwicklung neuer und wirksamer Impfstoffe nötig.

\section{Literatur}

1 Thomas G. Tuberculosis. WHO Fact Sheet N. 104. Geneva: WHO; 2007.

2 Gutierrez MC, et al. Ancient origin and gene mosaicism of the progenitor of Mycobacterium tuberculosis. PLoS Pathog. 2005;Sep(1(1)):e5.

3 Beja-Pereira A, et al. The origin of European cattle: evidence from modern and ancient DNA. Proc Nat Acad Sci U S Am. 2006;103(21):8113-8.

4 Nerlich AG, et al. Molecular evidence for tuberculosis in an ancient Egyptian mummy. Lancet. 1997;350(9088):1404.

5 Zimmerman MR. Pulmonary and osseous tuberculosis in an Egyptian mummy. Bull N Y Acad Med. 1979;55(6):604-8.

6 Cooper A, Poinar HN. Ancient DNA: do it right or not at all. Science. 2000;289(5482):1139.

7 Hippocrates. Epidemics. 400 BC.

8 Morton R. Phtisiologia, seu exercitationes de phtisi triubus libris comprehensae. Totumque opus variis hisoriis illustratum. London: Samuel Smith; 1689.

9 Schönlein JL. Zur Pathogenie der Impetigines. Arch Anat Physiol wissenschaftl Med. 1839:82.

10 Koch R. Die Aetiologie der Tuberculose. Berliner Klin Wochenschr. 1882;19(15):221-30. 
11 Rüttimann B. Volkssanatorien in der Schweiz. Schweiz Ärztezeitung. 2000;81(49):2821-4.

12 Trudeau EL. Environment in its relation to the progress of bacterial invasion in tuberculosis. Am J Med Soc. 1887;(84):118-23.

13 Finsen N. Om Lysets Indvirkningar paa Huden. Hospitalstidende. 1896;36:721-8.

14 Forlanini C. A Contribution to the Surgical Therapy of Phtisis. Ablation of the Lung? Artificial Pneumothorax? Gazetta degli Ospedali \& delle Cliniche die Milano. 1882;3:537.

15 Murray JF. Bill Dock and the location of pulmonary tuberculosis: how bed rest might have helped consumption. Am J Resp Crit Care Med. 2003; 168(9):1029-33.

16 Calmette A. La vaccination contre la Tuberculose. Paris: Masson; 1927.

17 Anonymous. The Lübeck Trial. Can Med Assoc J. 1932;26(3):362.

18 Tuberculosis Prevention Trial. Trial of BCG vaccines in south India for tuberculosis prevention: first report. Bull World Health Organ. 1979;57(5): 819-27.

19 Schatz AB, Waksman E. Streptomycin, a substance exhibiting antibiotic activity against Gram-positive and Gram-negative bacteria. Proc Soc Exp Biol Med. 1944;55:66-9.
20 Pfuetze KH, Feldman WH. Streptomycin in treatment of clinical tuberculosis. H. Corwin Hinshaw Papers. American Philosophical Society. 1946.

21 Pfuetze KH, et al. The first clinical trial of streptomycin in human tuberculosis. Am Rev Tuberculosis. 1955;71(5):752-4.

22 Mordasini E. 18 Monate Streptomycin in einer Tuberkulose-Heilstätte. Schweiz Med Wochenschr. 1948;78;605-12.

23 Gilbert D, et al. The Sanford Guide to Antimicrobial Therapy. 37th ed. Sanford Guide. 2007.

24 Helbling P. Tuberkulose in der Schweiz 2001-2004. BAG Bulletin. 2006:428-33.

25 Raviglione MC, Smith IM. XDR tuberculosis implications for global public health. N Eng J Med. 2007;356(7):656-9.

\section{Weiterführende Literatur}

- Dubos R, Dubos J. The White Plague: Tuberculosis, Man, and Society. Chapel Hill, NC: Rutgers University Press; 1952. 